\title{
CORRIGENDUM
}

\section{Telomerase inhibition, oligonucleotides, and clinical trials}

\author{
David R Corey
}

Oncogene (2002) 21, 6557. doi:10.1038/sj.onc.1205721

Correction to: Oncogene (2002) 21, 631-637.

doi:10.1038/sj.onc. 1205063

The authors wish to acknowledge support from the National Institutes of Health Lung Cancer SPORE program, grant P50CA70907. 